\title{
Experimental assessment of Taylor's hypothesis and its applicability to dissipation estimates in turbulent flows
}

\author{
Werner J. A. Dahm and Kenneth B. Southerland \\ Department of Aerospace Engineering, The University of Michigan, Ann Arbor, Michigan 48109-2118
}

(Received 29 October 1996; accepted 24 January 1997)

\begin{abstract}
Results are presented from an assessment of the applicability of Taylor's hypothesis for approximating streamwise derivatives and obtaining dissipation estimates in turbulent flows. These are based on fully resolved measurements of a conserved scalar field $\zeta(\mathbf{x}, t)$ throughout a four-dimensional spatio-temporal volume in a turbulent flow. The data allow simultaneous evaluation of all three components of the true gradient vector field $\boldsymbol{\nabla} \zeta(\mathbf{x}, t)$ and the time derivative field $(\partial / \partial t) \zeta(\mathbf{x}, t)$ at the small scales of a turbulent shear flow. Streamwise derivatives obtained from Taylor's frozen flow hypothesis yield a correlation of 0.74 with the true streamwise derivative field at the present measurement location in the self-similar far field of an axisymmetric turbulent jet. Direct assessments are also presented of approximations invoking Taylor's hypothesis to estimate energy dissipation rates in turbulent flows. The classical single-point time series approximation yields a correlation of 0.56 with the true scalar energy dissipation rate, while a mixed estimate that combines one spatial derivative and the time derivative gives a correlation of 0.72 . A general analytical formulation is presented for assessing various dissipation estimates, and for determining the optimal dissipation estimate that maximizes the correlation with the true dissipation rate. The resulting optimal mixed dissipation estimate yields a correlation of 0.82 at the point of maximum turbulence intensity in a jet, and a value of 0.92 on the jet centerline. (C) 1997 American Institute of Physics. [S1070-6631(97)01606-1]
\end{abstract}

\section{INTRODUCTION}

Since its introduction more than 50 yrs ago, Taylor's hypothesis ${ }^{1}$ has been widely used in measurements of gradient quantities in turbulent flows. The approximation, in which the local instantaneous value of the time derivative from a single-point measurement is used to estimate the spatial derivative in the streamwise direction, is routinely invoked to obtain gradient quantities when the required multipoint spatial measurements are impractical or otherwise unavailable. Indeed this approximation is used even under conditions well outside the range of validity originally envisioned by Taylor. He recognized that, in the limit of low turbulence intensities, the motion of gradients relative to the local mean flow could be approximated as one of pure convection. Under this assumption fluctuations of the quantity of interest, say $\zeta$, are considered frozen over the time scale of the temporal derivative, giving

$$
\left(\frac{\partial \zeta}{\partial x}\right)_{\mathrm{TH}} \equiv-\frac{1}{U}\left(\frac{\partial \zeta}{\partial t}\right),
$$

where $U$ is the local mean velocity along the streamwise direction, denoted here by $x$. In Taylor's studies of spectra in decaying wind tunnel turbulence, the underlying requirement for low turbulence intensity was well satisfied, and replacement of the spatial derivative by the time derivative was justified.

Taylor's original approximation has been invoked more liberally as a general means to estimate spatial derivatives in turbulent shear flows. Direct measurement of spatial velocity gradients (e.g., $\mathrm{Su}$ and $\mathrm{Dahm}^{2,3}$ ) as well as other gradient quantities in turbulent flows (e.g., Dahm, Southerland, and Buch; ${ }^{4}$ Southerland and Dahm $\left.{ }^{5,6}\right)$ is considerably more in- volved than the determination of temporal derivatives from single-point time series measurements. Consequently, owing to the importance of spatial gradient quantities in turbulence dynamics, it has been common to use Taylor's hypothesis to estimate spatial derivatives, even under conditions for which the approximation is not strictly valid. Even in multipoint probe measurements of velocity gradients (e.g., Vukoslavcevic, Wallace, and Balint; ${ }^{7}$ Tsinober, Kit, and Dracos ${ }^{8}$ ), Taylor's hypothesis is invoked to estimate derivatives along the mean streamwise direction. There have been numerous studies (e.g., Lin; ${ }^{9}$ Wygnanski and Fiedler; ${ }^{10}$ Antonia, PhanThien, and Chambers; ${ }^{11}$ Zaman and Hussain; ${ }^{12}$ Brown, Antonia, and Rajagopalan ${ }^{13}$ ) demonstrating errors introduced when making this approximation, and identifying alternative criteria under which the approximation may be justified in shear flows.

Among the most widespread uses of Taylor's hypothesis is in estimating dissipation rates in turbulent shear flows (e.g., Heskestad; ${ }^{14}$ Sreenivasan, Antonia, and Danh; ${ }^{15}$ Antonia, Phan-Thien, and Chambers, ${ }^{11}$ Brown, Antonia, and Rajagopalan; ${ }^{13}$ Anselmet and Antonia; ${ }^{16}$ Andrews et al. ${ }^{17}$ Dowling $\left.{ }^{18}\right)$. For any dynamically passive, conserved scalar quantity $\zeta(\mathbf{x}, t)$, the scalar gradient magnitude squared gives the dissipation rate $\chi(\mathbf{x}, t) \equiv-\nabla \zeta \cdot \nabla \zeta(\mathbf{x}, t)$ of the scalar energy $\frac{1}{2} \xi^{2}(\mathbf{x}, t)$ per unit mass of fluid, where the diffusivity has been absorbed in the normalization of the spatial coordinates. The true dissipation is thus

$$
\chi \equiv\left(\frac{\partial \zeta}{\partial x}\right)^{2}+\left(\frac{\partial \zeta}{\partial y}\right)^{2}+\left(\frac{\partial \zeta}{\partial z}\right)^{2}
$$

However, the simultaneous spatial derivatives required to evaluate $\chi(\mathbf{x}, t)$ have only recently become accessible to ex- 
perimental measurement, and for this reason various approximations for $\chi$ based on Taylor's hypothesis are generally used instead. The most common of these (e.g., Heskestad $;{ }^{14}$ Andrews et al. $;{ }^{17}$ Dowling ${ }^{18}$ ) uses Taylor's hypothesis in (1) to estimate the component of the gradient vector $\partial \zeta / \partial x$ along the mean streamwise direction, and then takes $\partial \zeta / \partial x=\partial \zeta / \partial y=\partial \zeta / \partial z$, giving the approximation

$$
\chi_{\mathrm{TH}} \equiv 3\left(-\frac{1}{U} \frac{\partial \zeta}{\partial t}\right)^{2} .
$$

In some cases, time series measurements at two closely spaced points (e.g., Anselmet and Antonia ${ }^{16}$ ) allow direct evaluation of one cross-stream spatial derivative component, in which case a mixed spatio-temporal dissipation approximation can be formed by combining the one available spatial derivative and the time derivative, as, for example,

$$
\chi_{2} \equiv\left(-\frac{1}{U} \frac{\partial \zeta}{\partial t}\right)^{2}+2\left(\frac{\partial \zeta}{\partial y}\right)^{2}
$$

While it has long been accepted that estimates of the type in (3) and (4) are only approximations to the true dissipation in turbulent shear flows, laboratory measurements necessary to assess the precise nature of the errors introduced have only recently become possible. The present results provide an assessment of Taylor's hypothesis in a turbulent shear flow using experimental data from four-dimensional spatio-temporal measurements of a conserved scalar field $\zeta(\mathbf{x}, t) \quad$ (Dahm, Southerland, and Buch; ${ }^{4}$ Southerland and Dahm; ${ }^{5,6} \mathrm{Su}$ and Dahm;, ${ }^{2,3}$ Frederiksen, Dahm, and Dowling ${ }^{19-21}$ ), each comprised of over three billion data points that extend simultaneously in all three spatial dimensions as well as time, and that resolve the smallest spatiotemporal gradients in the field. Particular emphasis is placed on evaluating the accuracy with which approximations based on Taylor's hypothesis in (1) can be used to estimate the true dissipation rate $\chi$ in (2) via $\chi_{\mathrm{TH}}$ and $\chi_{2}$ in (3) and (4). An analytical procedure for evaluating the relative merit of various dissipation estimates is presented and used to obtain an optimal two-point dissipation estimate that depends on the local turbulence intensity.

\section{MEASUREMENT TECHNIQUE}

The present results are from analyses of laser-induced fluorescence data for $S c \approx 2000$ mixing of a dynamically passive dye in the self-similar far field of an axisymmetric turbulent jet in water. The measurement technique was introduced by Dahm, Southerland, and Buch, ${ }^{4}$ and is described in detail by Southerland and Dahm. ${ }^{5,6}$ Briefly, the concentration field $\zeta(\mathbf{x}, t)$ of a laser fluorescent dye carried by the jet fluid was measured repeatedly in time within a small threedimensional spatial volume located 235 diam $(1.15 \mathrm{~m})$ downstream of the jet exit and $13 \mathrm{~cm}$ off the jet centerline. This radial location $(r / x=0.11)$ corresponds to the highest turbulence intensity in the jet, and thus provides a stringent test of Taylor's hypothesis. A highly collimated laser beam was swept in a raster fashion through this volume, and the resulting laser-induced fluorescence from dye-containing fluid was imaged onto a high-speed, planar, $256 \times 256$ ele- ment, photodiode array. The array output was serially acquired at eight-bits true digital depth and continuously written in real time to a 3.1 GB high-speed parallel-transfer disk bank capable of accommodating more than 50000 such $256^{2}$ data planes. The resulting measured fluorescence intensity field was subsequently converted to the true dye concentration. A typical two-dimensional data plane from a fourdimensional measurement is shown in Fig. 1.

Each measurement produces the scalar field at over three billion individual points in space and time. To estimate the resulting spatial and temporal resolution note that the local outer scale $\delta(x) \approx 0.44 x$ and the mean centerline velocity $u(x) \approx 7.2(J / \rho)^{1 / 2} x^{-1}$, with $J$ the jet source momentum flux and $\rho$ the ambient fluid density. At the outer scale Reynolds number $\operatorname{Re}_{\delta} \equiv(u \delta / \nu) \approx 3700$ and with the Schmidt number of 2075, the local strain-limited molecular diffusion length scale estimate is $\lambda_{\mathrm{D}} \approx 257 \mu \mathrm{m}$ and the local advection time scale estimate is $\tau_{D} \equiv \lambda_{\mathrm{D}} / u \approx 113 \mathrm{~ms}$. For comparison, the in-plane spatial resolution was $\Delta(x, y) \approx 109 \mu \mathrm{m}$. The (1/e) laser beam thickness was measured as $380 \mu \mathrm{m}$. Deconvolution of the scalar field measurements among adjacent planes increases the effective spatial resolution in the $z$ direction to the interplane separation, $\Delta z \approx 120 \mu \mathrm{m}$. These values show that both the characteristic scale of the pixel image volume $(\Delta x \cdot \Delta y \cdot \Delta z)^{1 / 3}$ and its maximum dimension $(\Delta z)$ are more than two times smaller than $\lambda_{\mathrm{D}}$. Similarly, the temporal separation between successive data planes was $\Delta t$ $=8.6 \mathrm{~ms}$, and comparing with the diffusion scale advection time of $113 \mathrm{~ms}$ verifies that the present measurements resolve essentially all of the fine scale structure of the local turbulent mixing process.

The separation in all three spatial dimensions between adjacent points within each spatial data volume is smaller than the local diffusion length scale $\lambda_{D}$ in the scalar field. This spatial resolution, together with the high signal quality attained, allows accurate differentiation of the measured conserved scalar field in all three spatial dimensions and in time. This makes it possible to determine the components of the true local instantaneous scalar gradient vector field $\boldsymbol{\nabla} \zeta(\mathbf{x}, t)$ throughout these four-dimensional data, without any need to resort to various approximations based on Taylor's hypothesis, as is commonly required. This in turn permits the determination of the true scalar energy dissipation rate field $\boldsymbol{\nabla} \zeta \cdot \boldsymbol{\nabla} \zeta(\mathbf{x}, t)$. Similarly, the temporal separation between adjacent data planes within each spatial data volume, and between the same data point in successive volumes in the fully four-dimensional data, is shorter than the local diffusion scale advection time $\tau_{D}$. As a result, it is possible to extract fully resolved time series data from both the conserved scalar field $\zeta(\mathbf{x}, t)$ and scalar energy dissipation rate field $\boldsymbol{\nabla} \zeta \cdot \boldsymbol{\nabla} \zeta(\mathbf{x}, t)$, again without invoking any of the various classical approximations. Moreover, note that $U \cdot \Delta t \approx \Delta x$, so that an accurate assessment of Taylor's hypothesis is indeed possible.

Finally, note that the imaged region in the turbulent scalar field in these experiments typically spans less than $\frac{1}{15}$ of the local outer scale $\delta$, and is comparable to the local inner scale $\lambda_{\nu}$ of the flow. The structure of velocity and scalar fields in turbulent shear flows at scales near and below $\lambda_{\nu}$ is 


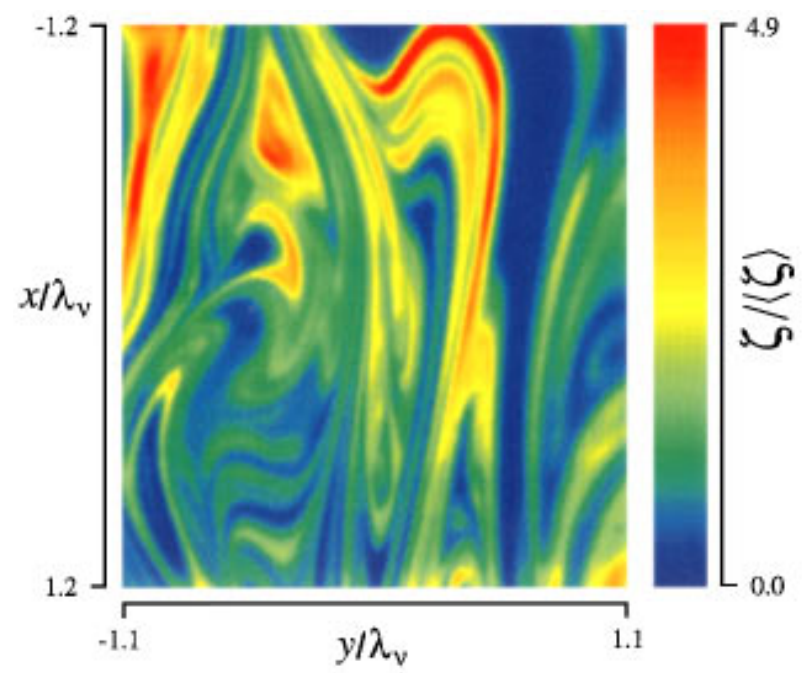

FIG. 1. Typical spatial data plane from the four-dimensional spatiotemporal data space $\zeta(\mathbf{x}, t)$ used in the present study. Spatial and temporal derivative results in this same plane are shown in Fig. 2, and comparisons of various dissipation estimates with the true dissipation rate field $\chi(\mathbf{x}, t) \equiv-D \nabla \zeta \cdot \nabla \zeta(\mathbf{x}, t)$ are shown in Fig. 4.

generally believed to be statistically universal. This contention appears to be true, even for the present moderate Reynolds number flows, as evidenced by the measured collapse of small-scale spectra at similar Reynolds numbers by Dowling, ${ }^{18}$ and by the DNS studies of Jiménez et al. ${ }^{22}$ The estimated Taylor scale Reynolds numbers for the present data are $38 \leqslant \operatorname{Re}_{\lambda} \leqslant 52$, well within the range of values over which the DNS results of Jiménez et al. showed Reynolds number-independent collapse on inner variables at the smallest flow scales. As a result, even though the present measurements are from $2900 \leqslant \operatorname{Re}_{\delta} \leqslant 5000$ turbulent jets, the smallscale structure and dynamics reflected in them should thus be largely representative of the inner scales of all turbulent shear flows.

\section{SPATIAL DERIVATIVES}

For the same data plane shown in Fig. 1, Fig. 2 compares the measured streamwise scalar field derivative values $(\partial \zeta / \partial x)$, in the appropriate inner scale variables, with the Taylor's hypothesis estimate $(\partial \zeta / \partial x)_{\mathrm{TH}}$ in (1) based on the measured time derivative values $(\partial \zeta / \partial t)$. Note that the color scales are identical in both cases. It can be clearly seen that, while the streamwise derivative estimate in the jet based on Taylor's hypothesis clearly captures the basic structure of the true streamwise derivative field, the approximation greatly overestimates large positive and negative values. This can be seen as well in the curves in Fig. 3, which show onedimensional intersections through fields of the type in Fig. 2. Note that Fig. 3(a) is a spatial intersection along the $y$ direction, while Fig. 3(b) is a temporal intersection. The profiles chosen in Fig. 3 are quite representative of the entire data space. The correlation between the two curves in Fig. 3(a) is 0.74 , and in Fig. 3(b) is 0.73 . For the entire data the correlation between $(\partial \zeta / \partial x)$ and $(\partial \zeta / \partial x)_{\mathrm{TH}}$ is found to be 0.74 . The correlation between the two planes in Fig. 2 is also 0.74, and thus representative of the entire data space. Notice that,

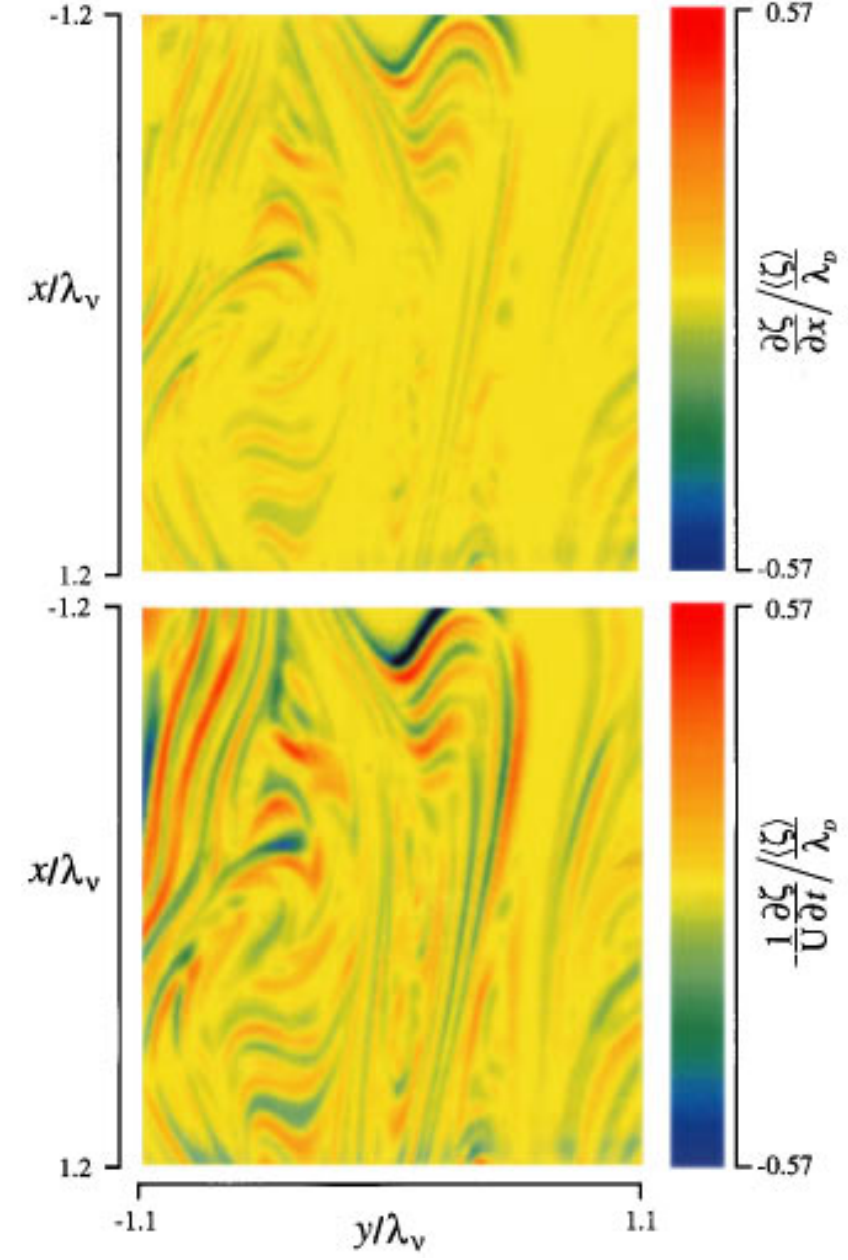

FIG. 2. Results showing typical comparison of the true streamwise derivative field $\partial \zeta / \partial x(t o p)$ with its Taylor's hypothesis approximation in terms of the time derivative $\partial \zeta / \partial t$ field (bottom). The correlation between these two fields is 0.74 .

as was suggested in Fig. 2, the extreme values are overestimated by the approximate derivative based on Taylor's hypothesis. As noted by Wygnanski and Fiedler, ${ }^{10}$ the comparatively large turbulence intensity in the jet far field, relative to the wind tunnel measurements for which Taylor's hypothesis was originally proposed, leads to significant errors in turbulence statistics when this hypothesis is used to estimate derivative quantities. Figures 2 and 3 give some insights into the nature of these errors.

\section{DISSIPATION ESTIMATES}

Figure 4 compares the true scalar energy dissipation rate $\chi$ in (2) with the approximation $\chi_{\mathrm{TH}}$ in (3) based solely on the time derivative in Fig. 2(b) and with $\chi_{2}$ from (4) based on mixed space and time derivatives. Results are shown both in linear form, where magnitudes can be readily compared, as well as in logarithmic form, where differences in the resulting dissipation field structure at low values can be better discerned. In each comparison, the color scales used for the true dissipation and the two approximations considered are identical, allowing direct comparisons. Note that while $\chi$, $\chi_{\mathrm{TH}}$, and $\chi_{2}$ all have the same fundamentally layer-like 

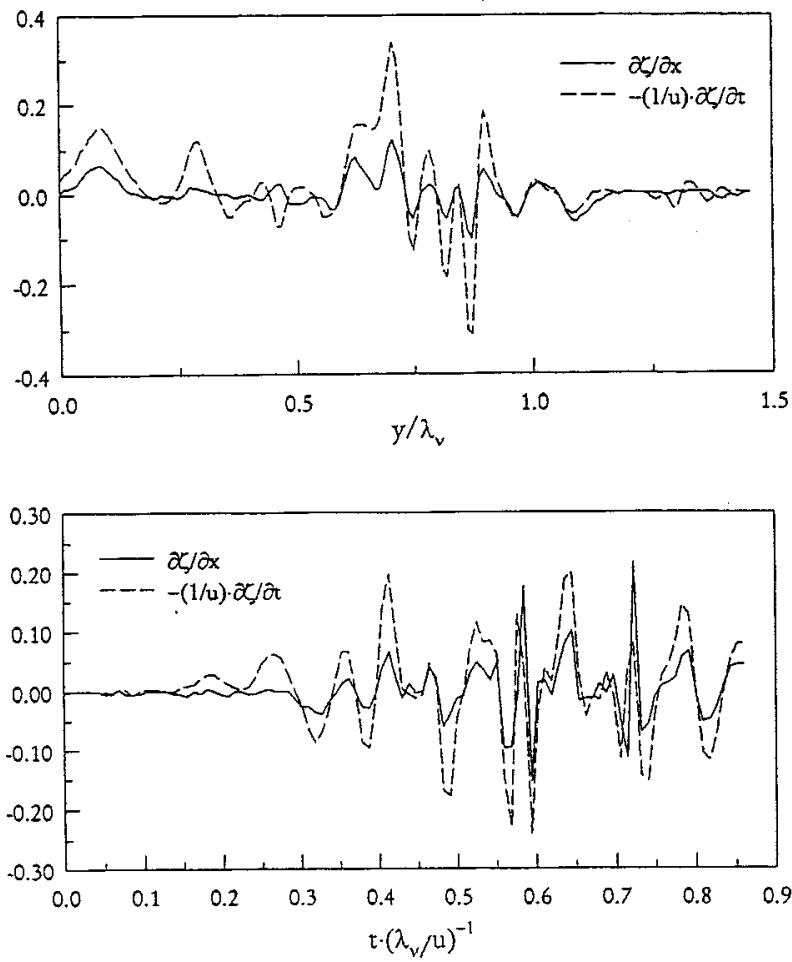

FIG. 3. Typical one-dimensional spatial (top) and temporal (bottom) intersections through the fully resolved four-dimensional data space, showing comparisons of the true streamwise derivative field $\partial \zeta / \partial x(\mathbf{x}, t)$ (solid line) with its Taylor's hypothesis approximation based on the time derivative field $\partial \zeta / \partial t(\mathbf{x}, t)$ (dashed line). The resulting correlation in (a) is 0.74 , and in (b) is 0.73 . The correlation obtained over the entire four-dimensional spatiotemporal data space is 0.74 .

character, considerable differences are apparent in the detailed structure of the dissipation fields. As would be expected from the exaggerated extreme values for the derivative fields in Figs. 2 and 3, the peak dissipation rates are greatly over represented in both the dissipation approximations in Fig. 4. Approximations similar to $\chi_{\mathrm{TH}}$ have been widely used in studies of the geometric scale similarity properties of the dissipation field in turbulent flows (see the discussion in Frederiksen, Dahm, and Dowling ${ }^{19-21}$ ). However, the results in Fig. 4 suggest that the differences between the true $\chi$ and $\chi_{\mathrm{TH}}$ can be rather large.

One-dimensional spatial intersections through the true dissipation field $\chi$ and the two approximate dissipation fields $\chi_{\mathrm{TH}}$ and $\chi_{2}$ are shown in Figs. 5(a) and 5(b). As was the case in Figs. 3(a) and 3(b), Fig. 5(a) is a spatial intersection along the $y$ direction, and Fig. 5(b) is a temporal intersection. While the intermittent character of the dissipation field is evident in all three cases, note that there are large differences apparent in the dissipation values. In some cases, even the dissipation support set is significantly different. In Fig. 5(a) the correlations between the true dissipation and the estimates $\chi_{\mathrm{TH}}$ and $\chi_{2}$ are 0.56 and 0.76, respectively, and in Fig. 5(b) are 0.52 and 0.79 . These are representative of the 0.56 and 0.72 correlation values obtained over the entire data space. As noted above, these differences are important for studies invoking Taylor's hypothesis to examine possible fractal and multifractal scaling from lower-dimensional ap- proximations of the true dissipation field (Frederiksen, Dahm, and Dowling ${ }^{19-21}$ ). It can also be seen in Figs. 4 and 5 that the dissipation estimates $\chi_{\mathrm{TH}}$ and $\chi_{2}$ tend to overestimate large dissipation rates. This is evident in Fig. 6, where the resulting distribution of dissipation values over the entire data space is shown for $\chi, \chi_{\mathrm{TH}}$, and $\chi_{2}$.

\section{ANALYTICAL CORRELATIONS}

For dissipation estimates like $\chi_{\mathrm{TH}}$ and $\chi_{2}$ in (3) and (4), it is possible to obtain analytical results for their correlations with the true dissipation $\chi$ in (2) under the assumption of isotropy in $\boldsymbol{\nabla} \zeta(\mathbf{x}, t)$. The correlation then allows an optimal dissipation estimate to be determined. The formulation makes use of the exact transport equation for the conserved scalar quantity $\zeta$, namely

$$
\frac{\partial \zeta}{\partial t}+\mathbf{u} \cdot \nabla \zeta-\frac{1}{\operatorname{ReSc}} \nabla^{2} \zeta=0
$$

where all variables have been normalized, giving the dimensionless diffusivity as the product $\mathrm{Re} \mathrm{Sc}$ of the Schmidt number and the Reynolds number based on the normalization scales. The second term in (5) can be written in terms of the scalar gradient vector magnitude $|\boldsymbol{\nabla} \zeta(\mathbf{x}, t)|$ and the projection $u_{\|}(\mathbf{x}, t) \equiv \mathbf{u} \cdot \hat{e}_{\nabla \zeta}$ of the velocity vector $\mathbf{u}(\mathbf{x}, t)$ onto the scalar gradient unit vector $\hat{e}_{\nabla \zeta}(\mathbf{x}, t)$, giving

$$
|\nabla \zeta(\mathbf{x}, t)|=-\frac{1}{u_{\|}}\left(\frac{\partial \zeta}{\partial t}-\frac{1}{\operatorname{ReSc}} \nabla^{2} \zeta\right) .
$$

Recognizing the diffusion term in (6) to be negligible at large Re Sc gives the gradient magnitude as

$$
|\nabla \zeta(\mathbf{x}, t)|=-\frac{1}{u_{\|}}\left(\frac{\partial \zeta}{\partial t}\right) .
$$

This should be compared with the classical Taylor's hypothesis for the streamwise derivative in (1). The true dissipation $\chi$ is simply the gradient magnitude squared, and from (7) is

$$
\chi=\left(-\frac{1}{u_{\|}} \frac{\partial \zeta}{\partial t}\right)^{2} .
$$

We first consider $\chi_{\mathrm{TH}}$ in (3) and note that

$$
\chi_{\mathrm{TH}}=3\left(\frac{u_{\|}}{U}\right)^{2} \chi .
$$

If statistical independence of $\mathbf{u}(\mathbf{x}, t)$ and $\boldsymbol{\nabla} \zeta(\mathbf{x}, t)$ is justified, then the correlation $R$ between $\chi$ and $\chi_{\mathrm{TH}}$ is simply

$$
R \equiv \frac{\overline{\chi_{\mathrm{TH}} \chi}}{\left(\overline{\overline{\mathrm{TH}}_{\mathrm{TH}}^{2}}\right)^{1 / 2}\left(\overline{\chi^{2}}\right)^{1 / 2}}=\frac{\overline{\left(u_{\|} / U\right)^{2}}}{\left[\overline{\left(u_{\|} / U\right)^{4}}\right]^{1 / 2}},
$$

namely the ratio of the second and fourth moments of $\left(u_{\|} / U\right)$. Since $\chi$ and $\chi_{\mathrm{TH}}$ in (9) are proportional via a statistically independent random factor, the absolute correlation in (10) and the conventional fluctuation correlation are equiva- 


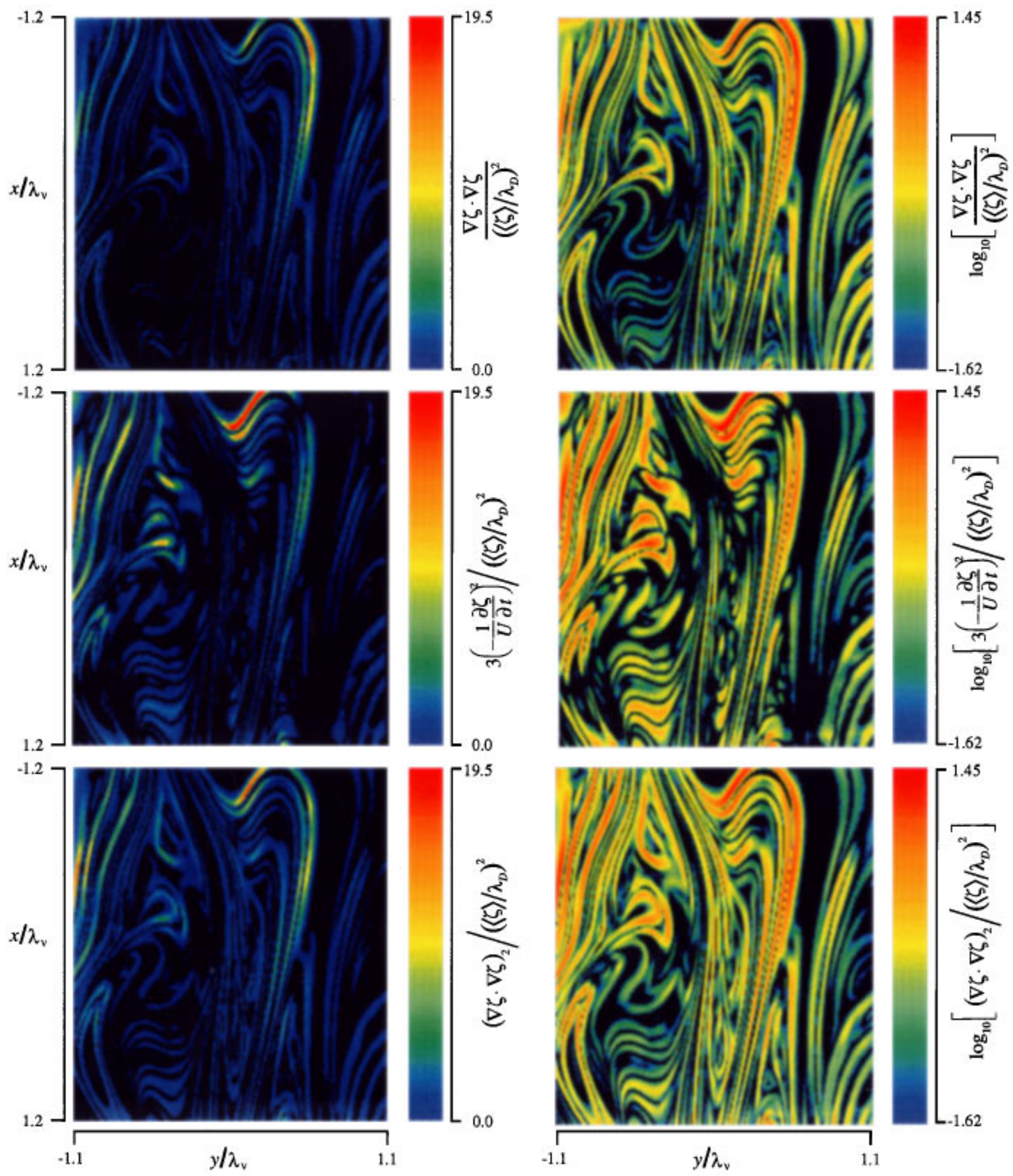

FIG. 4. Comparisons of the true dissipation rate field $\chi$ in (2) (top) with the single-point Taylor series approximation $\chi_{\mathrm{TH}}$ in (3) (middle) based solely on the time derivative in Fig. 2(b) and with the two-point mixed approximation $\chi_{2}$ in (4) (bottom) based on the time derivative and one spatial derivative. Results are in a linear form (left) to allow comparisons of relatively high dissipation rates, and in logarithmic form (right) allowing comparisons of lower values.

lent. Recognizing that $u_{\|} \equiv|\mathbf{u}| \cos \varphi$, where $\varphi$ is the spherical angle between $\mathbf{u}$ and $\boldsymbol{\nabla} \zeta$, and then taking $\boldsymbol{\nabla} \zeta$ to point with equal probability in all directions [with the isotropic distribution of spherical angles $\beta(\vartheta, \varphi)=(1 / 4 \pi) \sin \varphi]$, gives

$$
\overline{\left(\frac{u_{\|}}{U}\right)^{n}}=\frac{1}{n+1} \frac{\overline{\left(u^{2}+v^{2}+w^{2}\right)^{n / 2}}}{(\bar{u})^{n}},
$$

for $n$ even. Taking velocity fluctuations to be isotropic, and 

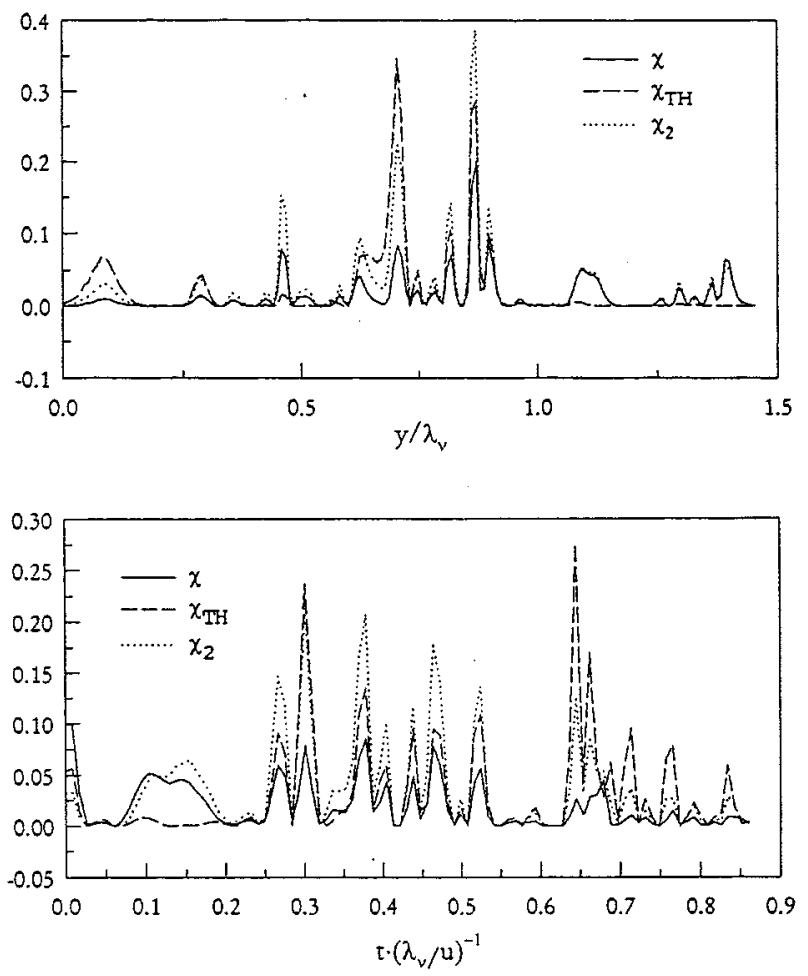

FIG. 5. Typical one-dimensional spatial (top) and temporal (bottom) intersections through the fully resolved four-dimensional data space, showing comparisons of the true scalar dissipation rate field $\chi(\mathbf{x}, t) \equiv-D \nabla \zeta \cdot \nabla \zeta(\mathbf{x}, t)$ in (2) (solid line) with the classical single-point Taylor's hypothesis approximation $\chi_{\mathrm{TH}}$ in (3) based on the time derivative field $\partial \zeta / \partial t(\mathbf{x}, t)$ (dashed line) and the mixed two-point approximation $\chi_{2}$ in (4) (dotted line). The correlation between $\chi$ and $\chi_{\mathrm{TH}}$ in (a) is 0.56 and in (b) is 0.52 , and over the entire four-dimensional spatio-temporal data space is 0.56 , which should be compared with the analytically predicted value of 0.60 in Sec. IV under the assumption of isotropy. Similarly, the correlation between $\chi$ and $\chi_{2}$ is 0.76 in (a) and 0.79 in (b), and 0.72 over the entire four-dimensional spatio-temporal data space, while the predicted value in $\mathrm{Sec}$ IV is 0.79 .

assuming Gaussian relations between fourth and second fluctuation moments (e.g., Millionshchikov, ${ }^{23,24}$ Monin and Yaglom $^{25}$ ), gives

$$
\begin{aligned}
& \overline{\left(\frac{u_{\|}}{U}\right)^{2}}=\frac{1}{3}\left[1+3\left(\overline{\frac{u^{\prime 2}}{\bar{u}^{2}}}\right)\right] \\
& \overline{\left(\frac{u_{\|}}{U}\right)^{4}}=\frac{1}{5}\left[1+10\left(\overline{\frac{u^{\prime 2}}{\bar{u}^{2}}}\right)+15\left(\overline{\frac{u^{\prime 2}}{\bar{u}^{2}}}\right)^{2}+12\left(\overline{\frac{u^{\prime} v^{\prime}}{\bar{u}^{2}}}\right)^{2}\right] .
\end{aligned}
$$

Profiles measured by Wygnanski and Fiedler ${ }^{10}$ for the mean velocity and turbulent stresses in the jet give the moments in (12a) and (12b) at $(r / x)=0.11$ as 0.71 and 1.41 , with the resulting correlation $R$ in (10) being 0.60 . This can be compared to the measured value of 0.56 obtained in Sec. IV, with the difference presumably due to departures from isotropy in $\boldsymbol{\nabla} \zeta$ (see Southerland and $\mathrm{Dahm}^{5,6}$ ) and the Gaussian relations in (12), and possibly from incomplete convergence of the velocity fluctuation statistics in (12) over the duration of the present scalar field measurements.

The same procedure can be applied for $\chi_{2}$ in (4), for which we note that

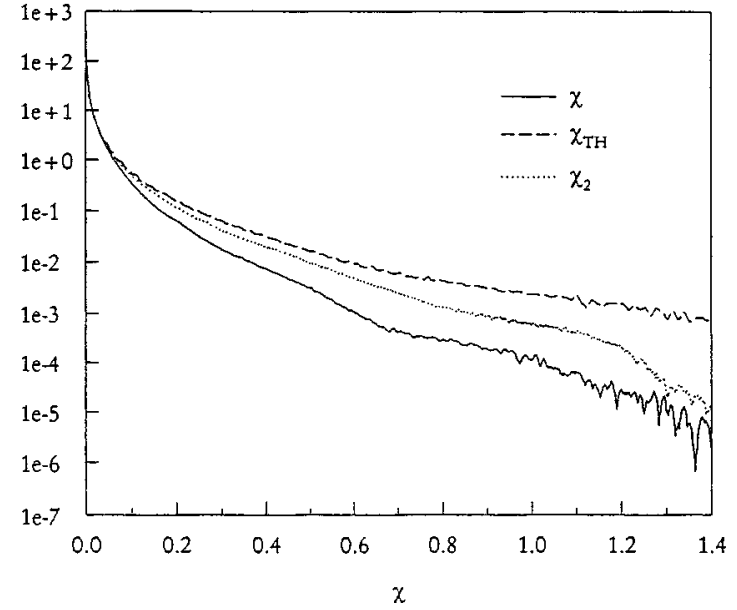

FIG. 6. Probability densities of the true dissipation rate $\chi$ (solid line), together with its single-point Taylor's hypothesis approximation $\chi_{\mathrm{TH}}$ in (3) (dashed line) and the mixed two-point approximation $\chi_{2}$ in (4) (dotted line). The approximations inherently overestimate the peak dissipation values, though $\chi_{2}$ represents a significant improvement over $\chi_{\mathrm{TH}}$. In Sec. IV we provide a general analytical formulation for constructing the optimal mixed two-point dissipation approximation.

$$
\chi_{2}=\left[\left(\frac{u_{\|}}{U}\right)^{2}+2 \frac{(\partial \zeta / \partial y)^{2}}{\chi}\right] \chi
$$

Recognizing that $(\partial \zeta / \partial y)=|\Delta \zeta| \cos \varphi$, with $\varphi$ now the spherical angle between $\nabla \zeta$ and $\hat{e}_{y}$, and again assuming isotropy in $\boldsymbol{\nabla} \zeta$ so that the distribution of spherical angles is $\beta(\vartheta, \varphi)=(1 / 4 \pi) \sin \varphi$, gives the correlation $R$ as

$$
R=\frac{\left[\overline{\left(u_{\|} / U\right)^{2}}+2 \overline{\cos ^{2} \varphi}\right]}{\left[\overline{\left(u_{\|} / U\right)^{4}}+4 \overline{\left(u_{\|} / U\right)^{2} \cos ^{2} \varphi}+4 \overline{\cos ^{4} \varphi}\right]^{1 / 2}} .
$$

Noting that the $n$th moment of $\cos \varphi$ is $1 /(n+1)$ for $n$ even, and using the moment values in (12) with the velocity fluctuation values from Wygnanski and Fiedler ${ }^{10}$ at the present $(r / x)=0.11$ location gives the correlation $R$ in (14) as 0.79 . This can be compared with the measured correlation 0.72 obtained in Sec. IV, with the difference again attributed to the factors mentioned above. In any case, the correlation of $\chi$ with $\chi_{2}$ is considerably higher than with $\chi_{\mathrm{TH}}$.

It is not surprising that the estimate $\chi_{\mathrm{TH}}$ based solely on Taylor's hypothesis is worse than $\chi_{2}$, which includes at least one measured component $(\partial \zeta / \partial y)^{2}$ of the true $\chi$. However, the same procedure for a $\chi$ estimate based purely on $3(\partial \zeta / \partial y)^{2}$ gives a correlation of only 0.74 . Thus the maximal correlation is achieved for a mixed dissipation estimate of the form

$$
\chi_{*} \equiv a\left(-\frac{1}{U} \frac{\partial \zeta}{\partial t}\right)^{2}+b\left(\frac{\partial \zeta}{\partial y}\right)^{2}
$$

for which

$$
R=\frac{\left[a \overline{\left(u_{\|} / U\right)^{2}}+b / 3\right]}{\left[a^{2} \overline{\left(u_{\|} / U\right)^{4}}+\frac{2}{3} a b \overline{\left(u_{\|} / U\right)^{2}}+b^{2} / 5\right]^{1 / 2}} .
$$

If the mean estimated dissipation is required to match the true mean $\chi$ then 


$$
a=\frac{3-b}{3 \overline{\left(u_{\|} / U\right)^{2}}} .
$$

The maximum correlation then results for

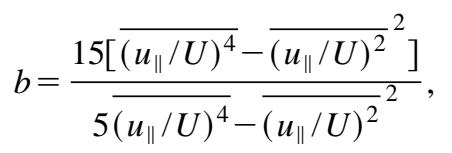

where the moments are given in (12). Again taking velocity fluctuation values in (12) from the measured turbulent jet profiles of Wygnanski and Fiédler ${ }^{10}$ gives the optimal $(a, b)$ values at the $(r / x)=0.11$ location of the present measurements as $a=0.51$ and $b=1.90$, and gives the correlation $R=0.82$. This differs only slightly from the value $0.79 \mathrm{ob}-$ tained above for $\chi_{2}$. However, this procedure is quite general and can be used to obtain the optimal two-point dissipation estimate at other locations in the jet and in other flows. For example, on the jet centerline the optimal values are $a=0.97$ and $b=1.80$, with the resulting correlation $R$ $=0.92$.

A similar approach may allow analogous results to be obtained for various estimates of the kinetic energy dissipation rate or other constructs obtained from the velocity gradient tensor components, and to obtain optimal estimates for these for any given measurement configuration or any given flow.

\section{CONCLUSIONS}

Fully resolved, four-dimensional, spatiotemporal laboratory data have provided an assessment of the errors made when Taylor's hypothesis is invoked to estimate spatial derivatives in a turbulent shear flow. The correlation of the streamwise gradient with its Taylor hypothesis estimate is found to be 0.74 at the present measurement location in the self-similar farfield of an axisymmetric turbulent jet. The simultaneous availability of accurate derivative information in all three spatial dimensions as well as time allows direct assessment of various approximations for the dissipation rate field based on implementations of Taylor's hypothesis. These show correlations with the true dissipation rate field of only 0.56 for $\chi_{\mathrm{TH}}$ in (3) and 0.72 for $\chi_{2}$ in (4). A general procedure introduced in Sec. VI, based on isotropy in the scalar and velocity fluctuations, allows the optimal dissipation estimate to be obtained for any given measurement configuration or any given flow.

\section{ACKNOWLEDGMENTS}

The measurements used for this study were obtained under support of the Airbreathing Combustion program of the Air Force Office of Scientific Research (AFOSR) under Grant No. AFOSR-89-0541 at Michigan.

${ }^{1}$ G. I. Taylor, “The spectrum of turbulence,'” Proc. R. Soc. London Ser. A 164, 476 (1938).
${ }^{2}$ L. K. Su and W. J. A. Dahm, "Scalar imaging velocimetry measurements of the velocity gradient tensor field in turbulent flows. I. Assessment of errors,"' Phys. Fluids 8, 1869 (1996).

${ }^{3}$ L. K. Su and W. J. A. Dahm, "Scalar imaging velocimetry measurements of the velocity gradient tensor field in turbulent flows. II. Experimental results,' Phys. Fluids 8, 1883 (1996).

${ }^{4}$ W. J. A. Dahm, K. B. Southerland, and K. A. Buch, "Direct, highresolution, four-dimensional measurements of the fine scale structure of Sc $\gg 1$ molecular mixing in turbulent flows," Phys. Fluids A 3, 1115 (1991).

${ }^{5}$ K. B. Southerland and W. J. A. Dahm, “A four-dimensional experimental study of conserved scalar mixing in turbulent flows," Report No. 02677912, Department of Aerospace Engineering, The University of Michigan, Ann Arbor, MI, 1994.

${ }^{6}$ K. B. Southerland and W. J. A. Dahm, "A four-dimensional experimental study of conserved scalar mixing in turbulent flows," submitted to J. Fluid Mech.

${ }^{7}$ P. Vukoslavcevic, J. M. Wallace, and J.-L. Balint, “The velocity and vorticity vector fields of a turbulent boundary layer. Part I. Simultaneous measurement by hot-wire anemometry," J. Fluid Mech. 228, 25 (1991).

${ }^{8}$ A. Tsinober, E. Kit, and T. Dracos, "Experimental investigation of the field of velocity gradients in turbulent flows," J. Fluid Mech. 242, 169 (1992).

${ }^{9}$ C. C. Lin, "On Taylor's hypothesis and the acceleration terms in the Navier-Stokes equations," Q. J. Appl. Math. 10, 295 (1953).

${ }^{10}$ I. Wygnanski and H. Fiedler, "Some measurements in the self-preserving jet," J. Fluid Mech. 38, 577 (1969).

${ }^{11}$ R. A. Antonia, N. Phan-Thien, and A. J. Chambers, "Taylor's hypothesis and the probability density functions of temporal velocity and temperature derivatives in a turbulent flow," J. Fluid Mech. 100, 193 (1980).

${ }^{12}$ K. B. M. O. Zamam and A. K. M. F. Hussain, "Taylor hypothesis and large-scale coherent structures,” J. Fluid Mech. 112, 379 (1981).

${ }^{13}$ L. W. B. Browne, R. A. Antonia, and S. Rajagopalan, "The spatial derivative of temperature in a turbulent flow and Taylor's hypothesis," Phys. Fluids 26, 1222 (1983).

${ }^{14} \mathrm{G}$. Heskestad, "A generalized Taylor's hypothesis with application for high Reynolds number turbulent shear flows," J. Appl. Math., Dec., 735 (1965).

${ }^{15}$ K. R. Sreenivasan, R. A. Antonia, and H. Q. Danh, "Temperature dissipation fluctuations in a turbulent boundary layer," Phys. Fluids 20, 1238 (1977).

${ }^{16} \mathrm{~F}$. Anselmet and R. A. Antonia, "Joint statistics between temperature and its dissipation in a turbulent jet," Phys. Fluids 28, 1048 (1985).

${ }^{17}$ L. C. Andrews, R. L. Phillips, B. K. Shivamoggi, J. K. Beck, and M. L. Joshi, "A statistical theory for the distribution of energy dissipation in intermittent turbulence,"’ Phys. Fluids A 1, 999 (1989).

${ }^{18}$ D. R. Dowling, "The estimated scalar dissipation rate in gas-phase turbulent jets," Phys. Fluids A 3, 2229 (1991).

${ }^{19}$ R. D. Frederiksen, W. J. A. Dahm, and D. R. Dowling, “Experimental assessment of fractal scale similarity in turbulent flows. Part I. Onedimensional intersections,"' J. Fluid Mech. 327, 35 (1996).

${ }^{20}$ R. D. Frederiksen, W. J. A. Dahm, and D. R. Dowling, "Experimental assessment of fractal scale similarity in turbulent flows. Part II. Higherdimensional intersections and nonfractal inclusions," J. Fluid Mech. 338, 89 (1997).

${ }^{21}$ R. D. Frederiksen, W. J. A. Dahm, and D. R. Dowling, "Experimental assessment of fractal scale similarity in turbulent flows. Part III. Multifractal scaling,', J. Fluid Mech. 338, 127 (1997).

${ }^{22}$ J. Jiménez, A. A. Wray, P. G. Saffman, and R. S. Rogallo, "The structure of intense vorticity in isotropic turbulence," J. Fluid Mech. 255, 65 (1993).

${ }^{23}$ M. D. Millionshchikov, "Theory of homogeneous isotropic turbulence," Dokl. Akad. Nauk. SSSR 22, 236 (1941).

${ }^{24}$ M. D. Millionshchikov, "Theory of homogeneous isotropic turbulence," Izv. Akad. Nauk. SSSR Ser. Georgr. Geofiz. 5, 433 (1941).

${ }^{25}$ A. S. Monin and A. M. Yaglom, Statistical Fluid Mechanics: Mechanics of Turbulence (MIT Press, Cambridge, 1975), Vol. 2. 